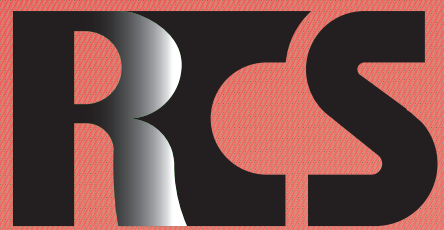

Depósito legal ppi $201502 Z U 4662$

Esta publicación científica en formato digital es continuidad de la revista impresa Depósito Legal: pp $197402 Z U 789$

- ISSN: 1315-9518 • ISSN-E: 2477-9431

Revista de Ciencias Sociales

Universidad del Zulia. Revista de la Facultad de Ciencias Económicas y Sociales Vol. XXVII. No. 2

Abril-Junio 2021

Esta publicación científica en formato digital es continuidad de la revista impresa Depósito Legal: pp $197402 Z$ Z789 ISSN: 1315-9518 


\title{
Plan de acompañamiento académico: Incidencia en el desarrollo de competencias genéricas en estudiantes universitarios
}

\author{
Castellar, Alex \\ Villadiego, Derlis** \\ Gamero, Harold ${ }^{* * *}$ \\ Gamarra, José ***** $^{\text {Gam }}$
}

\section{Resumen}

La educación como instrumento de desarrollo y formación del ser humano, persigue la intención fundamental de preparar profesionales integrales que respondan a las exigencias de la sociedad. En ese sentido, la Universidad de la Costa en Colombia, ha diseñado el Plan de Acompañamiento Académico con el propósito de mejorar las competencias de los estudiantes, es por ello, que el objetivo del presente articulo consiste en analizar la incidencia del Plan de Acompañamiento Académico sobre las competencias de lectura crítica y matemáticas, de los alumnos del primer semestre en dicha universidad. El abordaje metodológico fue empírico-analítico, cuantitativo, con diseño cuasi experimental pretest y postest. La muestra fue conformada por 171 estudiantes de pregrado. Los resultados evidencian la existencia de diferencias significativas al $95 \%$ (Wilcoxon W, p: .000) entre (pretest y postest), con un incremento en las competencias tanto de lectura crítica como de matemáticas, obteniéndose un mejoramiento cuantitativo del $78 \%$ en el nivel de desempeño de las matemáticas y un $90,7 \%$ en el de lectura crítica. En conclusión, estos hallazgos confirman la eficacia del Plan de Acompañamiento Académico para el mejoramiento en las competencias de lectura y matemáticas, permitiéndoles formarse como profesionales integrales y competitivos.

Palabras clave: Plan de acompañamiento académico; competencias genéricas; lectura crítica; matemáticas; educación superior.

\footnotetext{
Magister en Estadística Aplicada. Docente de la Universidad de la Costa, Colombia. E-mail: acastell6@cuc.edu.co (iD) ORCID: https://orcid.org/0000-0002-6637-3921

** Magister en Estadística Aplicada. Docente de la Universidad de la Costa, Colombia. E-mail: dvilladi3@cuc.edu.co iD ORCID: https://orcid.org/0000-0002-5221-6706

**** Magister en Ciencias Matemáticas. Docente de la Universidad del Atlántico, Colombia. E-mail: haroldgamero@mail. uniatlantico.edu.co iD ORCID: https://orcid.org/0000-0002-2016-6898

**** Magister en Ciencias Matemáticas. Docente de la Universidad ICESI, Colombia. E-mail: jose.gamarra@correo.icesi. edu.co iD ORCID: $\underline{\text { https://orcid.org/0000-0002-2906-9365 }}$
} 


\title{
Academic support plan: Incidence in the development of generic competences in university students
}

\begin{abstract}
Education as an instrument of development and training of the human being, pursues the fundamental intention of preparing integral professionals who respond to the demands of society. In this sense, the Universidad de la Costa in Colombia, has designed the Academic Accompaniment Plan with the purpose of improving the competencies of the students, which is why the objective of this article is to analyze the incidence of the Academic Accompaniment Plan on the critical reading and mathematics competencies of the students of the first semester at said university. The methodological approach was empirical-analytical, quantitative, with a quasi-experimental pretest and posttest design. The sample consisted of 171 undergraduate students. The results show the existence of significant differences at 95\% (Wilcoxon W, p: .000) between (pretest and posttest), with an increase in both critical reading and mathematics skills, obtaining a quantitative improvement of $78 \%$ in the performance level in mathematics and $90.7 \%$ in critical reading. In conclusion, these findings confirm the effectiveness of the Academic Accompaniment Plan for improving reading and math skills, allowing them to train as comprehensive and competitive professionals.
\end{abstract}

Keywords: Academic support plan; generic skills; critical reading; math; higher education.

\section{Introducción}

La educación como instrumento de desarrollo y formación del ser humano, tiene como propósito fundamental preparar profesionales integrales que respondan a las exigencias que la sociedad actual exige (Núñez-Peña, Bono y Suárez-Pellicioni, 2015; Lay, Ramírez y Parra, 2019; MartínezGarcés, Burbano-Vallejo y Burbano-Vallejo, 2019). Es por lo anterior, que las instituciones de educación superior tienen la gran responsabilidad de ofrecer a los jóvenes los escenarios en los que tengan la posibilidad de desarrollar competencias académicas, habilidades sociales, responsabilidad en el logro de metas, compromiso frente a la consecución de objetivos y formarse como profesionales competitivos, entre otras características, valores y actitudes que coadyuven en su formación integral, convirtiéndolos en profesionales altamente competentes (Carbonero, Román y Ferrer, 2013; Parra, et al., 2020).

Tomando como referente esta responsabilidad y en la búsqueda de alternativas que apunten a la permanencia y posterior graduación de sus estudiantes, la Universidad de la Costa, en Colombia, a través de la Vicerrectoría de Bienestar Universitario (Bienestar Universitario CUC, 2018), propone nivelar a los estudiantes que fueron admitidos en cualquier programa académico con un promedio por debajo de la media nacional en los resultados de las pruebas nacionales - Saber 11, específicamente en las competencias de lectura crítica y matemáticas. La competencia de lectura crítica, en el desarrollo universitario es de vital importancia para la producción de información y formación de la cognición (Vidal-Moscoso y Manríquez-López, 2016; Castro, Caicedo y Parra, 2017).

Así mismo, la competencia matemática ha sido estudiada por diversos autores debido al impacto en los estudiantes por el aplicativo de esta ciencia en la formación universitaria (Rodríguez-Muñiz y Díaz, 2015; Niebles, Martínez-Bustos y NieblesNúñez, 2020). El impacto de esta capacidad se debe principalmente a que la enseñanza 
de esta ciencia cambia drásticamente (Kajander y Lovric, 2005; Niebles, et al., 2020). Ambas competencias, son importantes para la formación integral del estudiante, contribuyendo así a la educación superior (Villarroel y Bruna, 2014; Casanova, et al., 2018).

En ese sentido, la Universidad de la Costa en Colombia, ha sido diseñado el Plan de Acompañamiento Académico (PAA) con el propósito de mejorar las competencias de esos estudiantes. Este proyecto, consta de un conjunto de guías teórico-prácticas en concordancia con las competencias en Lectura Crítica y Matemáticas exigidas por el Ministerio de Educación Nacional; los cuales corresponden en la competencia de lectura crítica, a los procesos de "Identificar y entender los contenidos explícitos de un texto, comprender cómo se articulan las partes de un texto para darle un sentido global y reflexionar a partir de un texto y evalúa su contenido" (Instituto Colombiano para la Evaluación de la Educación [ICFES], 2016a, p.4); mientras que para el área del razonamiento cuantitativo en lo referente a las matemáticas, conciernen a los procesos de "Interpretación y representación, Formulación y ejecución, y argumentación (ICFES, 2016b, p.2).

A partir de los planteamientos anteriores, surge el siguiente problema central: ¿Cómo incide el Plan de Acompañamiento Académico en el desarrollo de las competencias genéricas de lectura crítica y matemáticas en estudiantes de primer semestre de la Universidad de la Costa? La hipótesis de trabajo correspondiente se formula con los siguientes términos: Si el Plan de Acompañamiento Académico tiene incidencia en las competencias genéricas, entonces se encontrarán incrementos estadísticamente significativos en los niveles estimados de las competencias de lenguaje y matemáticas de los estudiantes.

En coherencia con el problema e hipótesis formulados, y con el fin de alcanzar el objetivo de analizar la incidencia del Plan de Acompañamiento Académico sobre las competencias de lectura crítica y matemáticas, se implementó un diseño cuasi experimental, el cual contó con la aplicación de un pretest online a través del Software de Valoración Académica, desarrollado para estimar los niveles de competencias en matemáticas y lectura crítica; posteriormente, se realizaron siete (7) sesiones de intervención del PAA con talleres y ejercicios académicos, que potencializan el desarrollo de las competencias. Finalmente, se aplicó el postest con el mismo Software como mecanismo de evaluación, con el fin de hacer un comparativo entre la conducta de entrada y la de salida, y de cómo fueron impactados los estudiantes en su rendimiento académico. El análisis de los datos se adelantó con el Software Estadístico SPSS ${ }^{\circledR}$ (Versión 22).

\section{Referentes teóricos y empíricos}

La transición del nivel secundario al nivel universitario implica un reto para los estudiantes, teniendo en cuenta los procesos adaptativos a los que hacen frente con relación a los nuevos contextos y también a las demandas propias del aprendizaje Duche, et al. (2020). Al respecto, Díaz (2008) afirma que:

El problema de la transición en la universidad es común para una diversidad de estudiantes. Los problemas originados en la transición pueden ser severos para jóvenes provenientes de comunas rurales y pertenecientes a estratos socioeconómicos bajos, ya que poseen condiciones económicas y sociales, en términos relativos, desventajosas al ingresar a una universidad o carrera que posee mayoritariamente estudiantes pertenecientes a los estratos superiores (p.70).

Por su parte, Losada, Carrozzi y Pérez (2015) sostienen que existe una alta tasa de deserción en el primer año de las carreras universitarias en general y particularmente elevadas en las carreras de Ingeniería. Las causas que se postulan son: La diferencia entre la formación previa de los ingresantes; las competencias y conocimientos, que se requieren para los estudios universitarios; y, la situación socioeconómica de los mismos 
(Pía, et al., 2016). En tal sentido, Obaya, Vargas y Delgadillo (2011) mencionan que las competencias se definen como aquellas entradas que hacen referencia a la capacidad individual demostrada para ejecutar; por ejemplo, la posesión del conocimiento, destrezas y características personales que se necesitan para satisfacer las demandas especiales o requerimientos de una situación particular.

Los modelos más consolidados en Australia, Canadá y los Estados Unidos han propuesto ocho competencias básicas, con las cuales se pueden agrupar diferentes habilidades. En su totalidad las competencias básicas o genéricas apoyan la práctica profesional, e internacionalmente se han aplicado en la educación y en los ambientes laborales.

En Colombia, el Instituto Colombiano para la Evaluación de la Educación (ICFES, 2017), menciona que concibe el objetivo de la educación como el desarrollo de determinadas competencias y, en consecuencia, a estas como el objeto de la evaluación. Dentro de las diferentes competencias que pueden desarrollarse a lo largo del proceso educativo se distingue entre competencias genéricas y no genéricas. Las competencias genéricas, son aquellas que resultan necesarios para el desempeño social, laboral y cívico de todo ciudadano. Las competencias no genéricas, son las propias de disciplinas particulares, que resultan indispensables para profesiones $u$ oficios específicos.

Las universidades a nivel global se han interesado en generar estrategias, encaminadas a favorecer la retención de los estudiantes durante sus primeros años de estudio (Carbonero, et al., 2013); entendiendo la retención, como un programa que comprende las acciones que desarrolla el aparato educativo en una institución, para garantizar el acompañamiento al estudiante durante su trayectoria académica, de tal manera que, pueda culminar de forma exitosa. Su objetivo es proporcionar las herramientas necesarias para la terminación de los diferentes ciclos $\mathrm{y}$ etapas en los tiempos establecidos, y adicionalmente, asegurar el conocimiento necesario y el desarrollo de competencias y actitudes indispensables para desenvolverse en la vida (Pineda-Báez, Pedraza-Ortiz y Moreno, 2011).

Entre las estrategias que se plantean las Instituciones de Educación Superior, buscan facilitar herramientas de apoyo a los estudiantes que ingresan con niveles bajos en algunas competencias genéricas. Sin embargo, León, Risco y Alarcón (2014) frente a las investigaciones en entornos de Educación Superior mencionan que: "Las estrategias de aprendizaje por los estudiantes afectan las metas de un modelo educativo" (p.1), por lo tanto, no solo basta con implementar herramientas de enseñanza, sino que también es necesario desarrollar herramientas y tácticas para el aprendizaje.

Dentro de las estrategias que favorecen la retención estudiantil, se encuentran los programas de servicios académicos orientados a enfrentar de manera prioritaria, aunque no siempre exclusiva, las falencias académicas de los estudiantes. Las tutorías, consejerías $\mathrm{y}$ asesorías forman parte de las iniciativas que se plantean para paliar problemas referidos a deficiencias de comprensión de lectura, procesos de escritura y desarrollo de pensamiento lógico-matemático (PinedaBáez, et al., 2011).

Otra de las estrategias que algunas universidades han considerado para apoyar a los estudiantes es el Plan de Acompañamiento, concebido como una estrategia importante e indispensable para fomentar el desarrollo de distintas competencias en el universitario de primer año (Olivares, et al., 2019); esta estrategia contribuye al desarrollo de muchas competencias y habilidades, como la resolución de conflictos, capacidad de reflexión y pensamiento crítico frente a los aprendizajes adquiridos, la capacidad para aplicar el aprendizaje a situaciones reales de la vida cotidiana, entre otros (Álvarez y Gonzáles, 2015).

El PAA de la Universidad de la Costa (Colombia), tiene como propósito ofrecer un curso nivelatorio, a los estudiantes que 
ingresaron a la universidad en el primer semestre y fueron admitidos en cualquier programa académico, con un promedio por debajo de la media nacional en las pruebas de Estado -Saber 11, en las áreas de lenguaje y matemáticas.

La lectura crítica (relacionado al área de lenguaje), es de vital importancia para el desarrollo óptimo, comprensión y producción de textos por parte de los estudiantes (Bean, 2011; Benavidez y Sierra, 2013; Flórez, 2016; Suárez, et al., 2020). Por otra parte, la competencia genérica de matemáticas, según Olivares, et al. (2018) resalta en la importancia de la transición entre la educación secundaria y la educación superior, por lo tanto, no solamente es un cambio de contexto, sino de reglas, metodología y modelo educativo, con nuevos retos en el desarrollo de las competencias y el fortalecimiento de los procesos de aprendizaje. Por lo cual, es importante diseñar estrategias que ayuden a nivelar competencias que no se logran desarrollar en la etapa secundaria y por lo tanto, representan un riesgo de deserción estudiantil (Losada, et al., 2007).

El presente estudio, responde a los lineamientos generales de las competencias exigidas por el Ministerio de Educación Nacional de Colombia (MEN, 2018), frente a los procesos asociados tanto con la competencia de la lectura crítica como con la competencia genérica de matemáticas, en las cuales los profesionales en formación deben aplicarlas en contexto con su disciplina. En tal sentido, los estudiantes deben fortalecer los procesos de identificar, comprender y transformar e integrar los textos con su correspondiente análisis crítico a nivel hipertextual, y por otra parte, analizar las representaciones de datos matemáticos en distintos formatos (textos, tablas, gráficas, diagramas, esquemas).

Asimismo, en relación a la formulación y ejecución, el estudiante plantea e implementa estrategias que lleven a soluciones adecuadas. Frente a un problema que involucre información cuantitativa y argumentación, el alumno valida procedimientos y estrategias matemáticas utilizadas para dar solución a problemas. En coherencia con lo anterior, el
Plan de Acompañamiento Académico adopta las competencias genéricas de lectura crítica y razonamiento cuantitativo, para lograr la nivelación de los estudiantes que ingresan a primer semestre.

\section{Metodología}

La investigación se abordó desde el paradigma empírico analítico, con enfoque cuantitativo, bajo un diseño cuasi experimental de pretest y postest. Al respecto, Hernández, Fernández y Baptista (2014) determinan que "el enfoque cuantitativo, utiliza la recolección de datos para probar hipótesis con base en la medición numérica y el análisis estadístico, con el fin de establecer pautas de comportamiento y probar teorías" (p.4). En la presente investigación, se analizan los resultados del pretest y postest en cada una de estas competencias, comparando el nivel de avance de una prueba respecto a la otra y corroborando si existe diferencias significativas entre las medianas de estas pruebas.

A los participantes se les aplicó una prueba previa, y una posterior a las intervenciones, tal como lo exige el diseño cuasi experimental; esto es, a un grupo se le aplica una prueba previa al estímulo o tratamiento experimental, después se le administra el tratamiento, y finalmente, se le aplica una prueba posterior al estímulo (Hernández, et al., 2014). En efecto, el objetivo del pretest diseñado en el Software de Evaluación Académica, consiste en diagnosticar el nivel de desempeño en el que se encuentran los estudiantes frente a las competencias genéricas de lectura y matemáticas, luego son sometidos a un estímulo, a lo que se le ha llamado intervenciones, y finalmente, se les aplica el postest dentro del mismo Software.

\subsection{Población y muestra}

La población estuvo representada por 464 estudiantes matriculados en la Universidad 
de la Costa y que fueron admitidos con Plan de Acompañamiento de acuerdo con el Reglamento estudiantil, artículo 33, Acuerdo 1261 de 2018 (Universidad de la Costa CUC, 2018) para el periodo 2016-II. Estos estudiantes obtuvieron desempeños por debajo de la media nacional en las pruebas de Estado - Saber 11. Para tal caso, 130 estudiantes del total de la población asistieron al plan de acompañamiento en lectura crítica correspondiente al 28\%; 162 al de matemáticas concerniente al $35 \%$ y 172 , el $37 \%$ que debían realizar los dos planes de acompañamiento (ver Gráfico I).

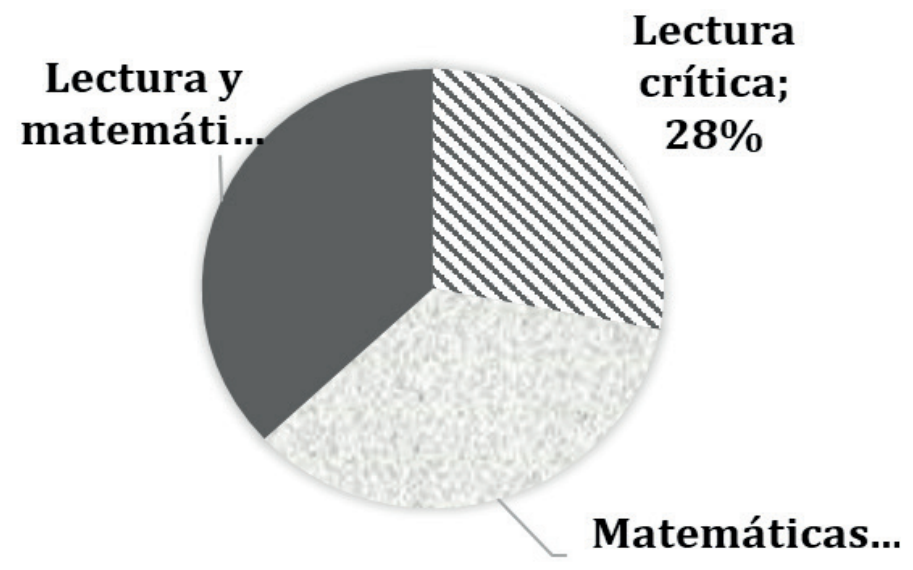

Fuente: Elaboración propia, 2021.

\section{Gráfico I: Estudiantes matriculados en el programa plan de acompañamiento} académico en el periodo 2016-II

De acuerdo con el planteamiento del problema y por acceso directo del investigador, se seleccionó una muestra no probabilística intencional de 171 estudiantes correspondientes al $37 \%$ del total de la población. Los participantes seleccionados estuvieron distribuidos de la siguiente manera: 80 estudiantes que asistieron al plan de acompañamiento en lectura crítica, 64 remitidos a Matemáticas y 27 que debían asistir paralelamente a los dos acompañamientos. En términos de porcentajes se puede apreciar en el Gráfico II. 


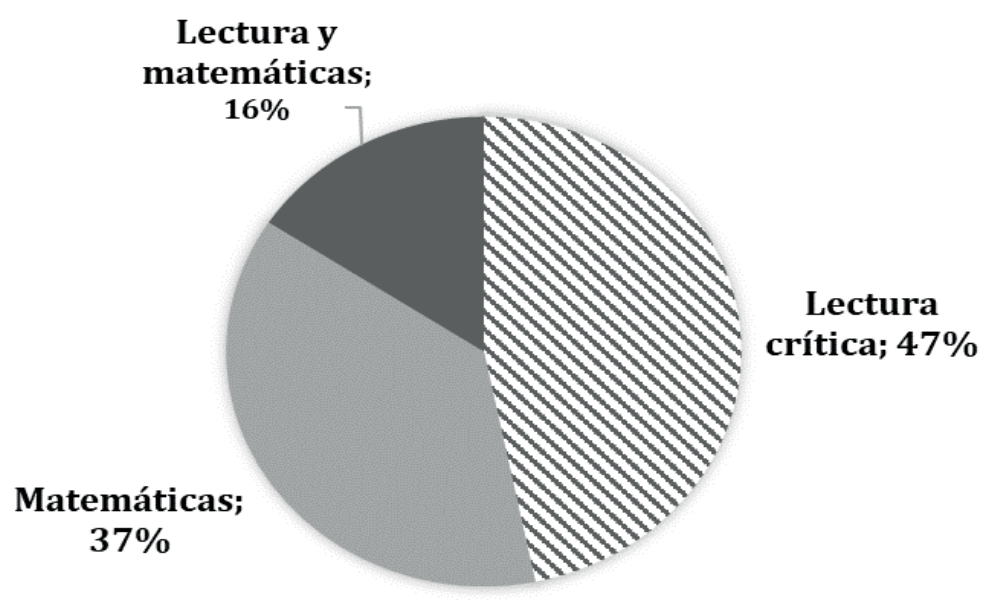

Fuente: Elaboración propia, 2021.

Gráfico II: Porcentaje de estudiantes de la muestra en plan de acompañamiento

Para lograr que esta muestra fuera homogénea se estandarizaron los siguientes criterios: a) Ser estudiante matriculado en un programa de pregrado en el periodo 2016 II; b) Haber obtenido un resultado por debajo de la media en las pruebas Saber 11, específicamente en las competencias de lectura y matemáticas; c) Estar matriculados en el plan de acompañamiento; $y, d$ ) Participar en las aplicaciones de tipo pretest y postest.

\subsection{Instrumentos}

Para la recolección de los datos se utilizaron los siguientes instrumentos:

\section{a. Reporte Estadístico Pruebas} Saber 11: Este instrumento arroja la información sobre puntajes que obtuvieron los estudiantes participantes de la investigación, específicamente en las competencias de lectura crítica y de matemáticas. Los datos utilizados en este trabajo se construyeron a partir de la información contenida en el sitio web del Instituto Colombiano, para la Evaluación de la
Educación Superior (ICFES).

b. Software de Valoración Académica, Versión 2.5: El mismo está parametrizado por dos pruebas, un pretest, que permite diagnosticar la conducta de entrada y un postest, que mide la conducta de salida. Luego, realiza un informe de tipo comparativo que mide el impacto de la intervención y el avance en los niveles de competencias genéricas en lectura crítica y matemáticas. Este software, permite la creación de $\mathrm{N}$ preguntas asociadas al tipo de prueba con sus respectivas respuestas, también consiente tener más de una pregunta correcta y tener varias incorrectas. El sistema (de manera aleatoria), selecciona una de las opciones de respuesta correcta, esto admite tener una aleatoriedad tanto en preguntas como en respuestas.

\subsection{Procedimiento}

La investigación se llevó a cabo en cinco (5) fases, las cuales se describen a continuación: 
a. Fase 1: Se prepararon los recursos de acceso al software y a la población. En esta fase, se caracterizan los participantes y se les da una inducción de acercamiento al software

b. Fase 2: Se recolectaron los datos del pretest (consentimiento informado) durante esta fase. Los docentes delegados de direccionar el curso, aplican la prueba diagnóstica (pretest) en una sala especializada con acceso al software. El estudiante ingresa sus datos personales en el mismo, una vez identificados, el sistema les permite acceder a la prueba. Cada prueba está constituida por 25 preguntas de selección múltiple con única respuesta que apuntan a identificar el nivel de competencias en que se encuentran los estudiantes. Los resultados del pretest, permiten corroborar cómo están los conocimientos previos de los participantes con respecto a las temáticas a trabajar durante el curso. El software proporciona clasificar en qué nivel de competencia se encuentran los estudiantes (bajo, medio, alto), tal como se puede observar en el Cuadro 1. Tanto el pretest como el postest fueron validados por expertos externos de la institución

\begin{tabular}{|ccc|}
\multicolumn{3}{c}{$\begin{array}{c}\text { Cuadro 1 } \\
\text { Resultados individuales evaluación pretest }\end{array}$} \\
\hline \multicolumn{1}{|c}{ Prueba } & $\begin{array}{c}\text { \% Preguntas } \\
\text { correctas }\end{array}$ & Nivel \\
\hline Lectura Critica & 40 & Bajo \\
Matemáticas & 20 & Bajo \\
\hline
\end{tabular}

Fuente: Elaboración propia, 2021.

c. Fase 3: Esta fase se llevó a cabo con la participación de profesores expertos para el desarrollo de cada competencia genérica (4 docentes para matemáticas y 3 para lectura crítica). Las intervenciones se desarrollaron en salones de 25 estudiantes en promedio, en horarios flexibles; el docente experto les suministra el material, explica el diseño y metodología de las guías y da las orientaciones pertinentes. Las intervenciones fueron diseñadas para un tiempo de 21 horas distribuidas en 7 sesiones de 3 horas semanales.

d. Fase 4: Una vez terminada las intervenciones y obtenidos los resultados evaluativos de los talleres, los participantes fueron convocados nuevamente a la sala especializada para la aplicación del postest. Esta prueba fue la misma que se le aplicó al inicio de la investigación (Fase 1); el objetivo de lo anterior es poder estimar un comparativo entre la conducta de entrada y la de salida, luego de haber sido intervenidos.

\section{Cuadro 2}

Resultados individuales evaluación postest

\begin{tabular}{lcc}
\hline \multicolumn{1}{c}{ Prueba } & \% Preguntas correctas & Nivel \\
\hline Lectura Critica & 56 & Medio \\
Matemáticas & 52 & Medio \\
\hline
\end{tabular}

Fuente: Elaboración propia, 2021. 
e. Fase 5: En esta fase se presentan los resultados obtenidos en la investigación, los cuales incluyen estadísticas descriptivas de algunas variables, así como un análisis estadístico comparativo entre datos no paramétricos con el uso de un software estadístico SPSS. Estos hallazgos se presentan y se discuten detalladamente en el siguiente apartado.

\section{Resultados y discusión}

Como primera instancia se procedió a realizar un análisis comparativo de los resultados arrojados por el pretest y el postest, de los desempeños evaluados a los estudiantes al finalizar cada prueba. Para el análisis de los resultados se utilizaron tres categorías de desempeño bajo (B), igual (I) y mejoró (M), los cuales representan el nivel de avance con respecto al resultado de la prueba pretest en relación con el resultado de la postest.

Se puede apreciar en la Tabla 1, que para el caso de la prueba de matemáticas sólo un $22 \%$ de los estudiantes (categoría bajo e igual), no mostraron evidencias de avances en sus competencias. En contraste al $78 \%$ que reflejó un aporte significativo, para una mejora en sus competencias matemáticas. $\mathrm{Al}$ respecto, se evaluaron: Interpretación y representación; formulación y ejecución; así como razonamiento y argumentación.

Tabla 1

Niveles de desempeño en matemáticas

\begin{tabular}{ccc} 
Categoría & Frecuencia & Porcentaje \\
\hline B & 13 & $14,3 \%$ \\
I & 7 & $7,7 \%$ \\
M & 71 & $78,0 \%$ \\
TOTAL & $\mathbf{9 1}$ & $\mathbf{1 0 0 , 0} \%$ \\
\hline
\end{tabular}

Fuente: Elaboración propia, 2021.

Para el caso de lectura crítica, se puede apreciar en la Tabla 2, que el 9,3\% de los estudiantes (categoría bajo e igual) no evidenciaron avances en sus competencias lectoras, frente a un 90,7\% de los estudiantes que lograron avanzar en sus niveles de lectura crítica. Es importante mencionar, que los estudiantes que presentaron en el comparativo de las pruebas pretest y postest un desempeño $\mathrm{B}$ e I, tienen como característica principal que no asistieron al total de las sesiones del Plan de Acompañamiento Académico, a diferencia de aquellos que acudieron a todas las sesiones, cumpliendo con el plan de trabajo establecido, estos estudiantes se encontraron en la categoría M. 
Tabla 2

Niveles de desempeño en lectura crítica

\begin{tabular}{ccc}
\hline Categoria & Frecuencia & Porcentaje \\
\hline B & 9 & $8 \%$ \\
I & 1 & $1 \%$ \\
M & 97 & $91 \%$ \\
TOTAL & $\mathbf{1 0 7}$ & $\mathbf{1 0 0 \%}$ \\
\hline
\end{tabular}

Fuente: Elaboración propia, 2021.

Los resultados fueron suministrados por el software de valoración en una escala de $0 \%$ a $100 \%$. Para realizar un análisis comparativo cualitativo, se estandarizaron los datos a una escala de 0.0 a 5.0 (sistema de calificación de la universidad) con la finalidad de hacer un análisis detallado del número de estudiantes que mejoraron luego del PAA. El análisis se realizó con las siguientes categorías: Categoría 1 (0.0 - 1.0); Categoría 2 (1.1 - 2.0); Categoría 3 (2.1 - 3.0); Categoría 4 (3.1 - 4.0); y, Categoría 5 (4.1 - 5.0).

El Gráfico III, permite evidenciar la cantidad de estudiantes que tuvieron notas entre 0.0 y 5.0 para la competencia de matemáticas. Por ejemplo, en el pretest 15 alumnos obtuvieron una nota inferior a 1.0, Sin embargo, luego de la intervención realizada, los resultados del postest muestran que solamente 3 estudiantes obtuvieron una calificación menor a 1.0. De igual manera, se observa que 24 estudiantes en el pretest obtuvieron una calificación entre 2.1 y 3.0 , sin embargo, el número de alumnos que obtuvieron esa calificación aumentó a 46.

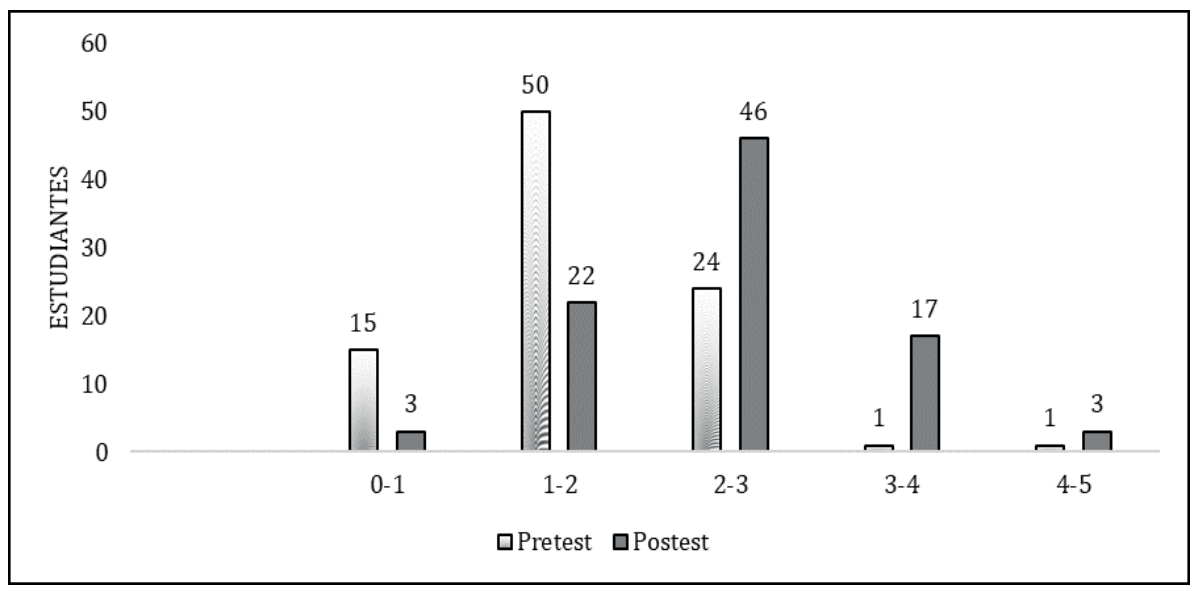

Fuente: Elaboración propia, 2021.

Gráfico III: Comparación entre resultados pretest y postest para las competencias de matemáticas 
Igualmente, el Gráfico IV permite evidenciar la cantidad de estudiantes que tuvieron notas entre 0.0 y 5.0 para la competencia de lectura. Por ejemplo, en el pretest 4 estudiantes obtuvieron una nota inferior a 1.0, Sin embargo, luego de la intervención realizada, los resultados del postest muestran que un estudiante obtuvo una calificación menor a 1.0. Así mismo, se observa que 0 estudiantes en el pretest obtuvieron una calificación entre 4.1 y 5.0, sin embargo, el número de estudiantes que obtuvieron esa calificación aumentó a 41 .

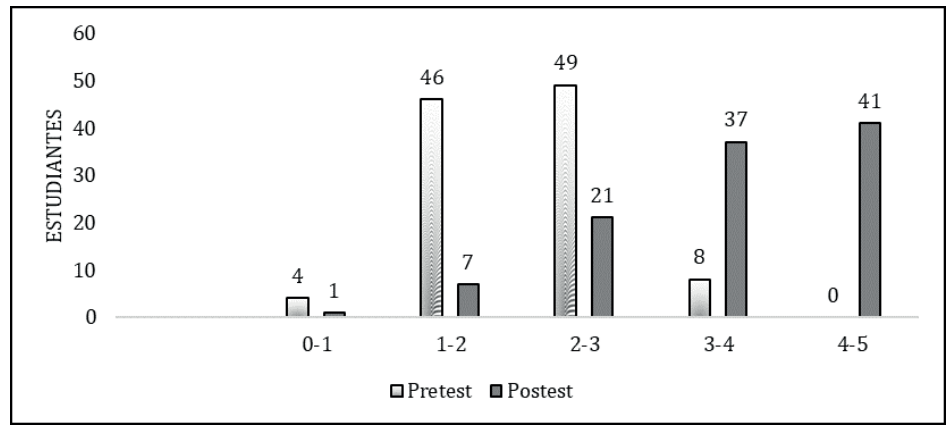

Fuente: Elaboración propia, 2021.

\section{Gráfico IV: Comparación entre resultados pretest y postest para las competencias de lectura}

Una vez realizado el análisis comparativo entre los resultados pretest $\mathrm{y}$ postest de valoración académica, se procedió a hacer un análisis de la distribución de los resultados para determinar el tipo de estadístico más adecuado. Primeramente, se examinó si los datos del pretest y postest de la prueba de valoración académica, tanto en matemáticas como en lectura crítica se ajustaban a la distribución normal. A través de la prueba Kolmogorov - Smirnov, tal como indican diferentes autores (Carbonero, et al., 2013; Visauta, 2018) con niveles de significación $(\mathrm{p}<0.05)$, por lo tanto, se optó por pruebas no paramétricas dado a la naturaleza de las observaciones (Ver Tabla 3).

\section{Tabla 3}

Resultados de la aplicación de la prueba de Kolmogorov-Smirnov para el pretest y postest de las competencias de matemáticas y lectura crítica

\begin{tabular}{cccc}
\hline & Estadístico & gl & p-valor. \\
\hline Pretest (matemáticas) & 0,131 & 91 & 0,001 \\
Postest (matemáticas) & 0,146 & 91 & 0 \\
Pretest (Lectura Crítica) & 0,203 & 107 & 0 \\
Postest (Lectura Crítica) & 0,116 & 107 & 0,001 \\
\hline
\end{tabular}

Fuente: Elaboración propia, 2021. 
Posteriormente, se aplicó la prueba no paramétrica de Wilcoxon (Carbonero, et al., 2013), con el fin de demostrar si existen diferencias entre los resultados de las pruebas del pretest y postest de los planes de acompañamiento en matemáticas y lectura crítica. En tal sentido, a continuación, en los
Cuadros 3 y 4 se presentan los resultados de la Prueba Wilcoxon, la cual permite evidenciar que existen diferencias significativas entre las pruebas $(\mathrm{p}<0.05)$, indicando la eficacia del plan de acompañamiento en el incremento de los niveles estimados de las competencias en lectura crítica y matemáticas.

\section{Cuadro 3}

\section{Prueba de Wilcoxon para comparar las medianas de las pruebas pretest y postest de matemáticas}

\begin{tabular}{llll}
\hline Hipótesis Nula & Prueba & Sig. & Decisión \\
\hline $\begin{array}{l}\text { La mediana de las diferencias entre } \\
\text { pretest } y \text { postest es igual a } 0\end{array}$ & $\begin{array}{l}\text { Prueba de rangos con signo } \\
\text { de Wilcoxon para muestras } \\
\text { relacionadas }\end{array}$ &, 000 & Rechazar la hipótesis nula \\
\hline
\end{tabular}

Nota: Se muestra significaciones asintóticas. El nivel de significación es de ,05.

Fuente: Elaboración propia, 2021.

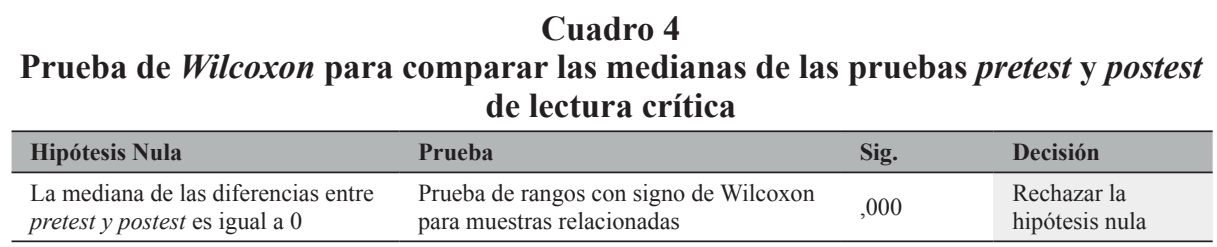

Nota: Se muestra significaciones asintóticas. El nivel de significación es de ,05.

Fuente: Elaboración propia, 2021.

Los hallazgos presentados, constituyen una evidencia relacionada con el impacto positivo del PAA, en el desarrollo de las competencias genéricas de matemáticas y lectura crítica de los estudiantes que participaron, quienes a su vez mejoraron su desempeño en las asignaturas del primer semestre. El incremento en los niveles de las competencias genéricas entre las puntuaciones pretest y postest, permitió adicionalmente, identificar que las actividades desarrolladas dentro del PAA, tales como: Desarrollo de guías de aprendizaje, talleres, ejercicios interactivos de resolución de problemas, planes de lectura, elaboración de escritos y asesoría individual, entre otros, contribuyen al desarrollo de las habilidades, competencias, hábitos y estilos de aprendizaje.

Tomando en cuenta lo anterior, se destaca que el desarrollo de competencias genéricas y transversales a las áreas del conocimiento, permiten mejorar el desempeño de los estudiantes (León, et al., 2014; Garcés y Mora, 2020). En la presente investigación se pudo evidenciar mediante pruebas no paramétricas de Wilcoxon, que existían diferencias significativas entre las pruebas pretest $\mathrm{y}$ postest. Estas pruebas permiten hacer un comparativo entre los resultados iniciales y finales, luego de haberse realizado un acompañamiento académico a los estudiantes de primer ingreso, esto con la finalidad de 
evidenciar el avance en las competencias genéricas de matemáticas y lectura crítica. Olivares, et al. (2018), en su investigación mencionan que hay una diferencia significativa favorable a nivel individual en las competencias. Estos resultados fueron evidenciables a través de una evaluación inicial y final (equivalencia a un pretest $\mathrm{y}$ postest respectivamente).

De igual manera, se pudo demostrar que la intervención a los estudiantes favorece al desarrollo de la competencia genérica de lectura por medio del PAA. Estos resultados posiblemente pueden estar ligados al desempeño en matemáticas, es decir, el mejoramiento de la competencia cognitiva de lectura puede favorecer el mejoramiento de la competencia cognitiva de matemáticas.

Lo anterior se sustenta bajo la afirmación de Flórez (2016), el cual establece: $\square$ la lectura en todos los niveles académicos es necesaria para el óptimo aprovechamiento de los alumnos, así como para el mejoramiento de su desempeño intelectual y cognitivo en todos los aspectos de la vida $\square$ (p.5). Es tan importante este campo cognitivo, que se han elaborado diferentes guías y libros para orientación en procesos de enseñanza. Por ejemplo, Bean (2011) en su libro $\square$ Ideas atractivas: la guía del profesor para integrar la escritura, el pensamiento crítico y el aprendizaje activo en el aula", establece cómo enfocar el pensamiento crítico en las diferentes disciplinas en relación con la teoría de la comunidad del género y el discurso.

A su vez, Benavidez y Sierra (2013), realizaron una investigación enfocada en la búsqueda de estrategias logísticas óptimas para la enseñanza y el aprendizaje de lectura crítica, obteniendo resultados de incidencia positiva sobre los estudiantes de la universidad EAN. Lo anterior, demuestra que el uso de instrumentos de enseñanza favorece al desarrollo de las competencias cognitivas, siendo el PAA una herramienta que impacta positivamente al desarrollo de la lectura crítica.

Con respecto a las competencias de matemáticas, Rodríguez-Muñiz y Díaz (2015), afirman que el hecho de que un estudiante realice un curso cero de matemáticas (similar a un PAA) no es motivo para afirmar que ellos rindan más que aquellos estudiantes que no lo hicieron. En contraste, la presente investigación permitió visualizar cuantitativamente un avance significativo al realizar un PAA, esto es evidenciable al analizar los resultados pretest - postest.

\section{Conclusiones}

Dentro de las principales conclusiones obtenidas derivadas de los hallazgos, se encontró con el análisis de las diferentes bases de datos, evidencias con la finalidad de plantear que el acompañamiento académico orientado a desarrollar competencias genéricas en matemáticas y lectura crítica, es pertinente para nivelar a estudiantes de primer ingreso que presentan dificultades detectadas desde la prueba nacional de Estado.

De igual manera, esta investigación permitió afirmar que la aplicación del PAA de la Universidad de la Costa en Colombia, tiene una incidencia positiva en los estudiantes que fueron evaluados mediante del software de valoración académica para las competencias de lectura crítica y matemáticas. En tal sentido, el análisis estadístico permitió demostrar que hubo un mejoramiento cuantitativo del $78 \%$ en el nivel de desempeño de matemáticas y un $90,7 \%$ en el desempeño de lectura crítica, estableciendo que las intervenciones aplicadas a los estudiantes tuvieron gran impacto en el mejoramiento del desempeño de los alumnos evaluados.

\section{Referencias bibliográficas}

Álvarez, P. R., y Gonzáles, M. C. (2015). Estrategias de intervención tutorial en la universidad: Una experiencia para la formación integral del alumnado de nuevo ingreso. Tendencias Pedagógicas, 16, 237-256. 
Castro, G., Caicedo, M., y Parra, J. C. (2017). Factores asociados a la adquisición de competencias en América Latina. Revista de Ciencias Sociales (Ve), XXIII(4), 33-52.

Bean, J. C. (2011). Engaging ideas: The professor's guide to integrating writing, critical thinking, and active learning in the classroom. JosseyBass.

Benavidez, D. R., y Sierra, G. M. (2013). Estrategias didácticas para fomentar la lectura crítica desde la perspectiva de la transversalidad. REICE. Revista Iberoamericana sobre Calidad, Eficacia y Cambio en Educación, 11(3), 79-109.

Bienestar Universitario CUC (2018). Paspe. Nuestros servicios. CUC. http:// bienestar.cuc.edu.co/

Carbonero, M. A., Román, J. M., y Ferrer, M. (2013). Programa para "aprender estratégicamente" con estudiantes universitarios: Diseño y validación experimental. Anales de Psicología, 29(3), 876-885. $\quad$ https://doi. org/10.6018/analesps.29.3.165671

Casanova, I., Canquiz, L., Paredes, I., e Inciarte, A. (2018). Visión general del enfoque por competencias en Latinoamérica. Revista de Ciencias Sociales (Ve), XXIV(4), 114-125.

Díaz, C. (2008). Modelo conceptual para la deserción estudiantil Universitaria Chilena. Estudios Pedagógicos (Valdivia), $\quad X X X I V(2), \quad 65-86$. https://doi.org/10.4067/S0718$\underline{07052008000200004}$

Duche, A. B., Paredes, F. M., Gutiérrez, O. A., y Carcausto, L. C. (2020). Transición secundaria-universidad y la adaptación a la vida universitaria. Revista de Ciencias Sociales (Ve), XXVI(3), 244258. $\quad$ https://doi.org/10.31876/rcs. v26i3.33245
Flórez, D. (2016). La importancia e impacto de la lectura, redacción y pensamiento crítico en la educación superior. Zona Próxima, (24), 128-135.

Garcés, J., y Mora, C. (2020). Estrategias de aprendizaje para mitigar la deserción estudiantil en el marco de la COVID-19. SUMMA. Revista Disciplinaria en Ciencias Económicas y Sociales, 2(E), 49-55.

Hernández, R., Fernández, C., y Baptista, M. D. P. (2014). Metodología de la investigación. Editorial McGraw-Hill.

Instituto Colombiano Para la Evaluación de la Educación - ICFES (2016a). Módulo de Lectura Crítica: Pruebas Saber Pro 2016-2. https://www.icfes.gov.co/ documents/20143/494634/Guia $\% 20$ de $\% 20$ orientacion $\% 20$ modulo $\% 20$ lectura $\% 20$ critica $\% 20$ saber $\% 20$ pro $\% 202016 \% 202$.pdf

Instituto Colombiano Para la Evaluación de la Educación - ICFES (2016b). Módulo de Razonamiento Cuantitativo: Pruebas Saber Pro 2016-2. https://www.icfes. gov.co/documents/20143/494634/ Guia $\% 20$ de $\% 20$ orientacion $\% 20$ modulo\%20razonamiento $\% 20$ cuantitativo $\% 20$ saber $\% 20$ pro $\% 20$ 2016\%202.pdf

Instituto Colombiano Para la Evaluación de la Educación - ICFES (2017). Saber 11. https://www.icfes.gov.co/ documents/20143/177687/Guia+ de+orientacion+saber+11-2017-1. pdf/b694a2e9-a61d-3e9c-1f00$\underline{\text { b3a9a576a767 }}$

Kajander, A., y Lovric, M. (2005). Transition from secondary to tertiary mathematics: McMaster University experience. International Journal of Mathematical Education in Science and Technology, 36(2-3), 149-160. https://doi. org/10.1080/00207340412317040

Lay, N., Ramírez, J., y Parra, M. (2019). 
Desarrollo de conductas ciudadanas en estudiantes del octavo grado de una institución educativa de Barranquilla. En R. López, Memorias del I Congreso Internacional en Educación e Innovación en Educación Superior. Caracas, Venezuela. Fondo Editorial UPEL.

León, A. P., Risco, E., y Alarcón, C. (2014). Estrategias de aprendizaje en educación superior en un modelo curricular por competencias. Revista de la Educación Superior, 43(172), 123-144.

Losada, M. A., Carrozzi, L. E., y Pérez, M. V. (Octubre 2015). La persistencia en los estudiantes de la FCA-UNMDP. El caso de la COHORTE 2007 en la carrera Ingeniería Agronómica. Studylib.Es. VIII Jornadas Nacionales y I Congreso internacional sobre la Formación del Profesorado, Mar de la Plata, Argentina.

Martínez-Garcés, J., Burbano-Vallejo, M., y Burbano-Vallejo, E. (2019). Obstáculos y perspectivas al emplear tecnologías de información para enseñar contabilidad. Educación y Humanismo, 21(37), 104-119. https:// doi.org/10.17081/eduhum.21.37.3461

Ministerio de Educación Nacional de Colombia - MEN (2018). Conozca los aportes a la definición de las Competencias Genéricas en la Educación Superior. MEN. https://www.mineducacion.gov. co/1759/w3-article-189357.html? noredirect $=1$

Niebles, W., Martínez-Bustos, P., y NieblesNúñez, L. (2020). Competencias matemáticas como factor de éxito en la prueba pro en universidades de Barranquilla, Colombia. Educación y Humanismo, 22(38), 1-16. https://doi. org/10.17081/eduhum.22.38.3590

Núñez-Peña, M. I., Bono, R., y SuárezPellicioni, M. (2015). Evaluación formativa en Educación Superior: Impacto en estudiantes con ansiedad a las matemáticas. Procedia - Social and Behavioral Sciences, 196, 135-141. https://doi.org/10.1016/j. sbspro.2015.07.023

Obaya, A., Vargas, Y. M., y Delgadillo, G. (2011). Aspectos relevantes de la educación basada en competencias para la formación profesional. Educación Química, 22(1), 63-68.

Olivares, S. L., Adame, E., Avila, J. E., Turrubiates, M. L., López, M. V., y Valdez-García, J. E. (2019). Valor percibido de una experiencia de inmersión educativa para el desarrollo de competencias trasversales: Semana i. Educación Médica, 20(S2), 93-99. https://doi.org/10.1016/j. edumed.2018.04.015

Parra, M., Marambio, C., Ramírez, J., Suárez, D., y Herrera, H. (2020). Educational convergence with digital technology: Integrating a global society. In $22^{\text {nd }}$ International Conference on HumanComputer Interaction (pp. 303-310). Springer, Cham.

Pía, A., Díaz, M. C., Pauletti, C., y Scarimbolo, D. (2016). Experiencia de un curso nivelatorio de matemática para la cohorte 2015 de la carrera de ingeniería agronómica de la UNLPam. REPEM, VI, 254-264. http://redi. exactas.unlpam.edu.ar/xmlui/ bitstream/handle/2013/223/CB23\%20 -\%20Andrea $\% 20$ PIA.pdf? sequence $=1$

Pineda-Báez, C., Pedraza-Ortiz, A., y Moreno, I. D. (2011). Efectividad de las estrategias de retención universitaria: La función del docente. Educación y Educadores, 14(1), 119-135.

Rodríguez-Muñiz, L. J., y Díaz, P. (2015). Estrategias de las universidades españolas para mejorar el rendimiento en matemáticas del alumnado de nuevo ingreso. Aula Abierta, 43(2), 
69-76. $\quad$ https://doi.org/10.1016/j. aula.2015.01.002

Suárez, N., Pérez, I. C., Rodríguez, A., y Sevilla, S. (2020). Lectura crítica en el desarrollo de habilidades de investigación en profesores de postgrado. Revista de Ciencias Sociales (Ve), XXVI(E-2), 328339. https://doi.org/10.31876/rcs. v26i0.34131

Universidad de la Costa CUC (2018). Reglamento Estudiantil. Universidad de la Costa CUC. Acuerdo No. 1261, 29 de Octubre de 2018. https://bit. ly/3aSZeQL

Vidal-Moscoso, D., y Manríquez-López, L. (2016). El docente como mediador de la comprensión lectora en universitarios. Revista de la Educación Superior, 45(177), 95-118. https://doi. org/10.1016/j.resu.2016.01.009

Villarroel, V., y Bruna, D. (2014). Reflexiones en torno a las competencias genéricas en educación superior: Un desafío pendiente. Psicoperspectivas. Individuo y Sociedad, 13(1), 2334-34. $\quad$ https://doi.org/10.5027/ psicoperspectivas-Vol13-Issue 1fulltext-335

Visauta, B. (2018). Análisis estadístico con SPSS para Windows: Estadística básica. SERBIULA. 